\title{
THE SURFACE TENSION OF THE BLOOD SERUM IN HYPERTHYROIDISM
}

By ELIZABETH G. NICHOLLS AND GEORGE A. HARROP, JR.

(From the Chemical Division of the Medical Clinic of the Johns Hopkins University and Hospital, Baltimore)

(Received for publication November 8, 1927)

The present communication is a report of an investigation of the surface tension of the blood serum in patients with severe thyroid intoxication. The general plan of the study has been as follows: Determinations of the surface tension were made upon samples of blood serum from untreated patients immediately upon admission to the hospital. Treatment with iodine (in the form of Lugol's solution) was then given in sufficient amount and over a sufficient period of time to allow an exhibition of its clinical effect, when the surface tension studies were repeated. Later the surface tension was again determined after surgical operation (double partial lobectomy of subtotal thyroidectomy) in order to observe any change resulting from the diminished activity of the thyroid gland due to its partial. removal. In some cases iodine was continued for a short time after operation.

The direct reading tensiometer of Du Noüy (1) was used for making the surface tension determinations, and all the precautions recommended by him were carefully followed. The watch glasses used were of uniform size (diameter $8 \mathrm{~cm}$.) and the same amount of serum, approximately $2 \mathrm{cc}$., used each time. The glassware used was boiled for two hours in a concentrated solution of sulphuric acid to which had been added $15 \mathrm{cc}$. saturated solution of potassium bichromate per liter. The watch glasses were washed not longer than two or three days before using and were flamed a short time before use in order to insure uniform spreading of the serum. The greatest care was taken throughout to avoid touching with the hands any glassware, including the collecting apparatus, centrifuge tubes and watch glasses.

The blood for the surface tension determinations was collected in 
TABLE 1

Surface tension determinations

\begin{tabular}{|c|c|c|c|c|c|c|}
\hline \multirow{2}{*}{ Subject } & \multirow[b]{2}{*}{ Date } & \multicolumn{3}{|c|}{$\begin{array}{l}\text { Surface tension- } \\
\text { serum }\end{array}$} & & \multirow{2}{*}{ Remarks } \\
\hline & & $\begin{array}{c}\text { Initial } \\
\text { read- } \\
\text { ing }\end{array}$ & $\begin{array}{c}\text { Read- } \\
\text { ing } \\
\text { after } \\
2 \text { hours }\end{array}$ & Drop & & \\
\hline \multicolumn{7}{|c|}{ Normal subjects } \\
\hline \multirow{5}{*}{ E. G. N. } & & $\left|\begin{array}{c}\text { dymes } \\
\text { per } \\
\text { sq. cm, }\end{array}\right|$ & $\begin{array}{c}\text { dymes } \\
\text { per } \\
\text { sq. cm. }\end{array}$ & dynes & \multirow{5}{*}{ Staff } & \\
\hline & November 18,1925 & 56.6 & 49.6 & 7.0 & & \\
\hline & November 27, 1925 & 56.8 & 49.7 & 7.1 & & \\
\hline & January 7, 1926 & 57.1 & 49.6 & 7.5 & & \\
\hline & January 8, 1926 & 56.7 & 49.6 & 7.1 & & \\
\hline \multirow[t]{2}{*}{ G. A. H. } & November 18, 1925 & 56.6 & 49.7 & 6.9 & Staff & \\
\hline & November 27, 1925 & 56.6 & 49.6 & 7.0 & Staff & \\
\hline \multirow[t]{2}{*}{ E. H. } & November 18, 1925 & 56.8 & 49.4 & 7.4 & Staff & \\
\hline & December 1, 1925 & 56.6 & 49.3 & 7.3 & Staff & \\
\hline T. G. & November 18, 1925 & 57.1 & 49.8 & 7.3 & Staff & \\
\hline A. $S$. & January 15, 1926 & 57.1 & 49.7 & 7.4 & Student & \\
\hline H. P. & January 15,1926 & 56.8 & 49.8 & 7.0 & Student & \\
\hline C. W. & January 20, 1926 & 57.1 & 49.5 & 7.6 & Student & \\
\hline I. T. . & January 20, 1926 & 56.9 & 49.3 & 7.6 & Student & \\
\hline S. W. & January 20, 1926 & 56.9 & 49.3 & 7.6 & Student & \\
\hline L. W. & January 20, 1926 & 57.5 & 49.3 & 8.2 & Student & \\
\hline E. T. & January 20, 1926 & 57.2 & 49.1 & 8.1 & Student & \\
\hline W. S. & January 22, 1926 & 57.0 & 49.1 & 7.9 & Student & \\
\hline J. B. & February 2, 1926 & 56.8 & 49.9 & 6.9 & Student & \\
\hline E. C. & February 2, 1926 & 57.7 & 49.8 & 7.9 & Student & \\
\hline
\end{tabular}

Miscellaneous conditions

\begin{tabular}{|c|c|c|c|c|c|}
\hline G. C. & November 10,1925 & 57.6 & 49.8 & 7.8 & $\begin{array}{l}\text { Incipient menopause; psycho- } \\
\text { neurosis }\end{array}$ \\
\hline R. C. & November 10, 1925 & 57.1 & 49.4 & 7.7 & $\begin{array}{l}\text { Bronchial asthma, very mild re- } \\
\text { action to ragweed and feathers }\end{array}$ \\
\hline C. $\mathbf{T}$. & November 10, 1925 & 56.6 & 49.5 & 7.1 & $\begin{array}{l}\text { Unexplained vertigo. Diag- } \\
\text { nosis: disease of vestibularap- } \\
\text { paratus following fracture of } \\
\text { skull in } 1921\end{array}$ \\
\hline F. P. & November 10, 1925 & 57.1 & 49.6 & 7.5 & $\begin{array}{l}\text { Pregnancy, second month (de- } \\
\text { livery normal) }\end{array}$ \\
\hline P. L. & $\begin{array}{l}\text { January 27, } 1926 \\
\text { February 2, } 1926\end{array}$ & $\begin{array}{l}56.5 \\
56.7\end{array}$ & $\begin{array}{l}49.5 \\
49.5\end{array}$ & $\begin{array}{l}7.0 \\
7.2\end{array}$ & Hysteria \\
\hline
\end{tabular}


TABLE 1-Concluded

\begin{tabular}{|c|c|c|c|c|c|}
\hline \multirow[b]{2}{*}{ Subject } & \multirow[b]{2}{*}{ Date } & \multicolumn{3}{|c|}{$\begin{array}{l}\text { Surface tension- } \\
\text { serum }\end{array}$} & \multirow[b]{2}{*}{ Remarks } \\
\hline & & $\begin{array}{c}\text { Initial } \\
\text { read- } \\
\text { ing }\end{array}$ & $\begin{array}{c}\text { Read- } \\
\text { ing } \\
\text { after } \\
2 \text { hours }\end{array}$ & Drop & \\
\hline \multicolumn{6}{|c|}{ Miscellaneous conditions-continued } \\
\hline & & $\left|\begin{array}{c}\text { dynes } \\
\text { per } \\
\text { sq. cm. }\end{array}\right|$ & $\begin{array}{c}\text { dymes } \\
\text { per } \\
\text { sq. cm. }\end{array}$ & dymes & \\
\hline F. B. & February 2, 1926 & 56.9 & 49.4 & 7.5 & Palsy \\
\hline R. S. & January 14, 1926 & 57.5 & 49.5 & 8.0 & Arthritis deformans \\
\hline \multirow[t]{2}{*}{ F. Mc. } & January 14, 1926 & 57.0 & 49.9 & 7.1 & Arthritis deformans \\
\hline & January 21, 1926 & 57.1 & 49.9 & 7.2 & Arthritis deformans \\
\hline M. M. & December 24, 1925 & 56.9 & \begin{tabular}{l|l}
9 & 49.2
\end{tabular} & 7.7 & Recovered coryza \\
\hline G. C. & January 18, 1926 & 57.3 & $3 \mid 49.5$ & 7.8 & Convalescent pneumonia \\
\hline \multirow{2}{*}{ C. J. } & January 14, 1926 & 56.7 & 7) 49.6 & 7.1 & Scleroderma \\
\hline & January 27, 1926 & 56.6 & \begin{tabular}{l|l}
6 & 49.4
\end{tabular} & 7.2 & Scleroderma \\
\hline O. N. & January 22, 1926 & 57.0 & 48.8 & 8.2 & Scleroderma \\
\hline
\end{tabular}

the morning after sixteen hours of complete fasting, by venapuncture without stasis. It was drawn directly into a $15 \mathrm{cc}$. centrifuge tube through an L-shaped glass capillary tube ground to fit on a platinum needle. After clotting it was centrifuged and the serum was poured into a watch glass, stirred, and a reading immediately made. After the reading, the watch glass containing the serum was covered by an inverted Petri dish and allowed to stand undisturbed for two hours, when a second reading was made to ascertain the time drop. In order to avoid disturbing the serum while standing, the watch glasses were placed on a revolving table similar to that described by $\mathrm{Du}$ Noüy.

All readings were made between $23^{\circ}$ and $24^{\circ} \mathrm{C}$. by placing the apparatus in a hood where the temperature could be regulated and air currents eliminated. A control reading of the surface tension of running tap water was made at the same time as that of the serum to be sure the instrument was accurately adjusted. Tap water was preferred to distilled water because of the extreme care needed for proper preparation of the latter. Undiluted serum was used because it required little handling and the chance of contamination was therefore 


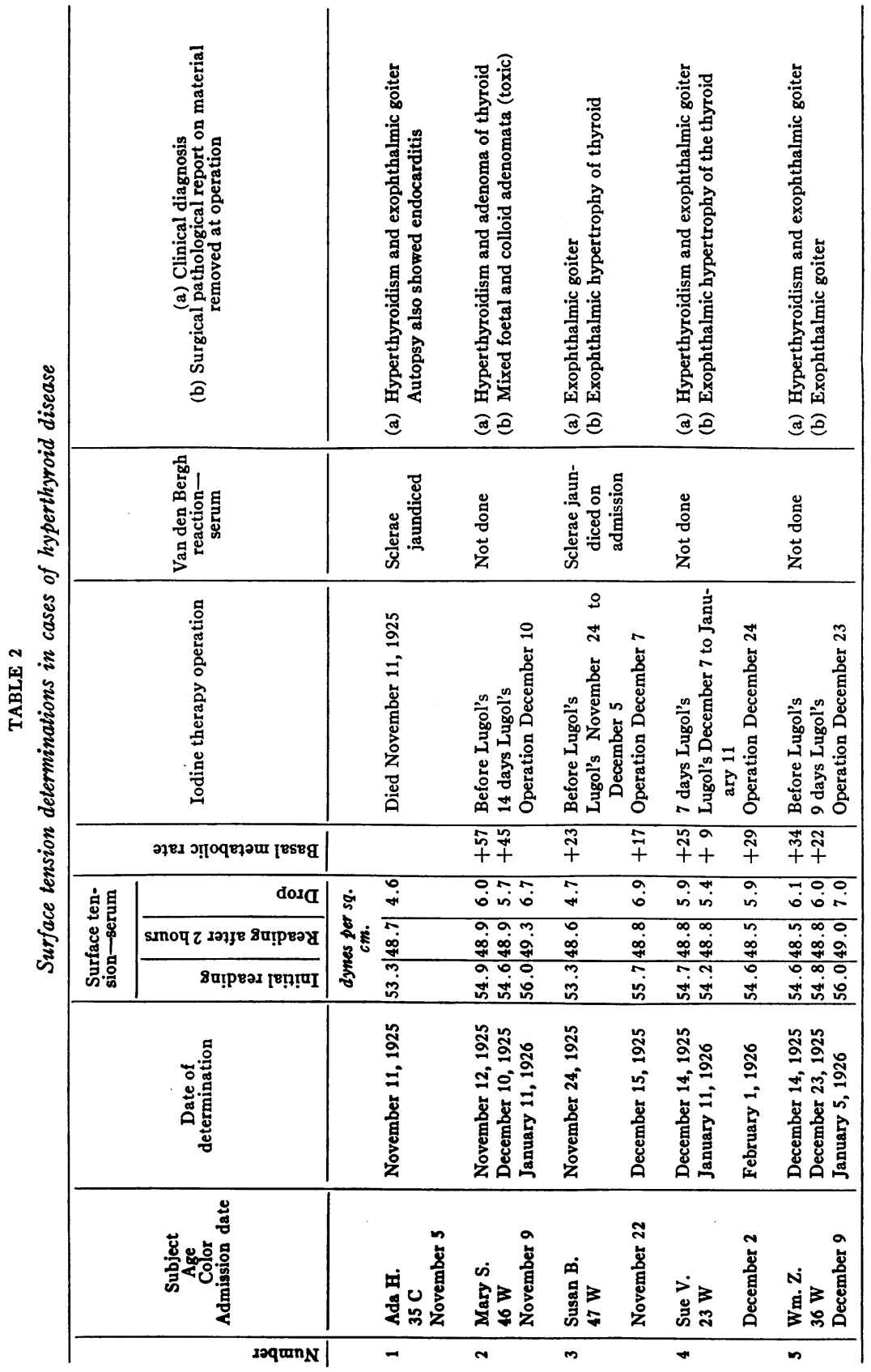


E. G. NICHOLLS AND G. A. HARROP, JR.

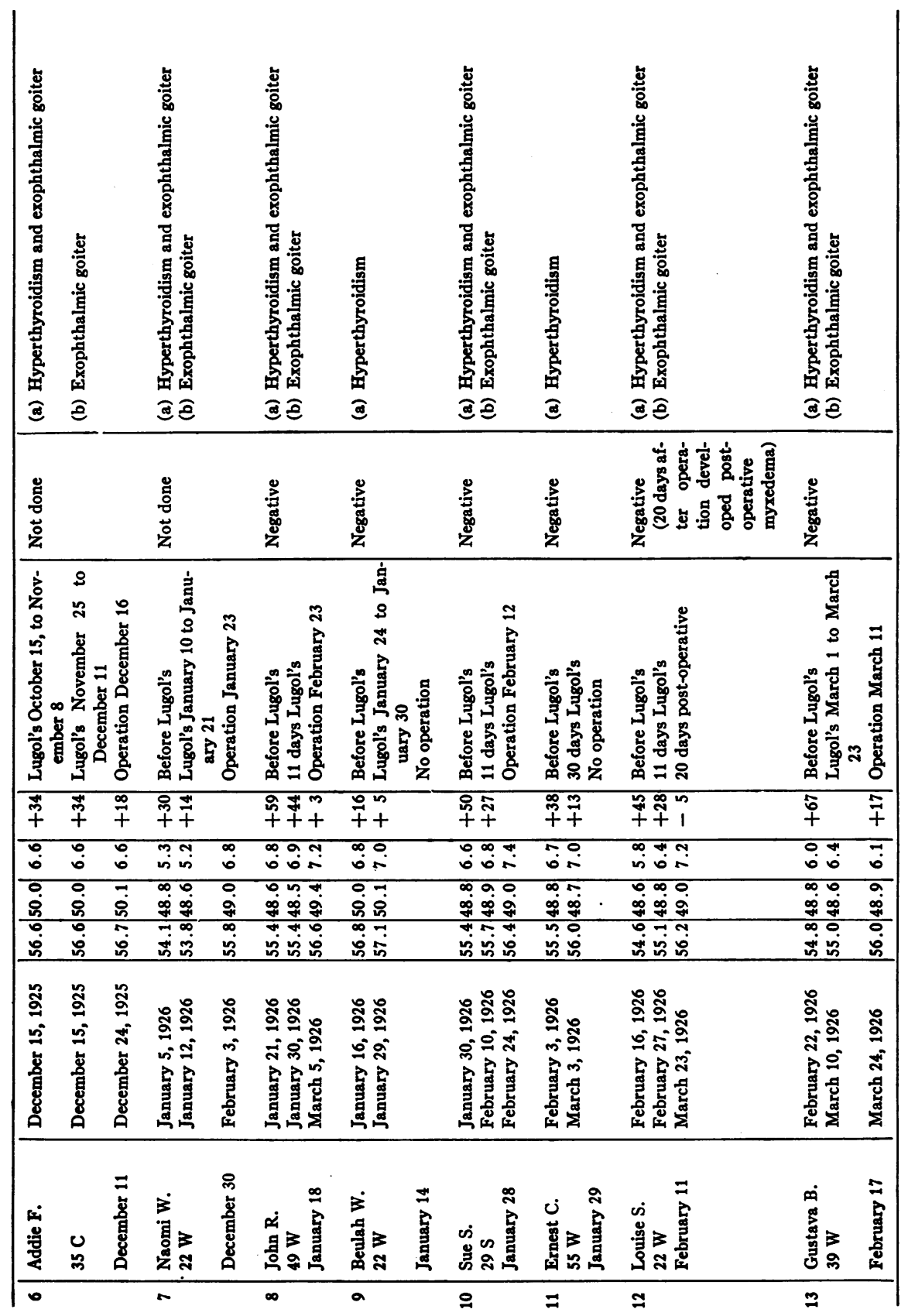




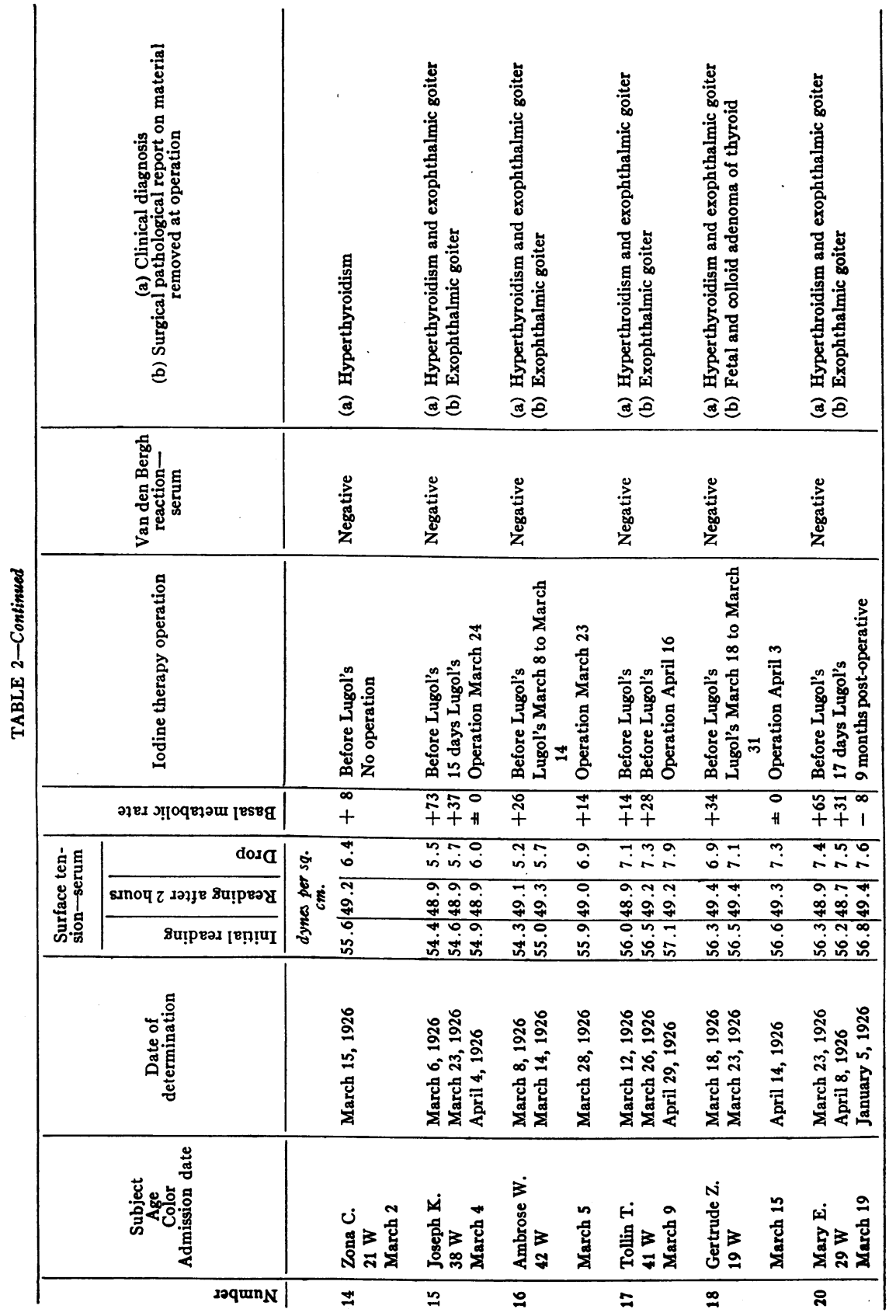


E. G. NICHOLLS AND G. A. HARROP, JR.

\begin{tabular}{|c|c|c|c|c|c|c|c|c|c|c|}
\hline 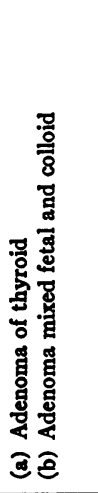 & 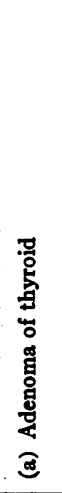 & 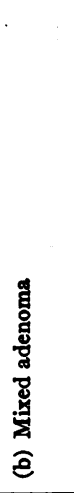 & 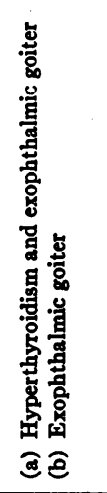 & 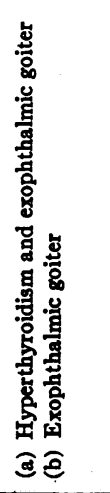 & 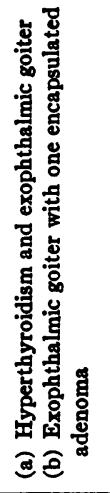 & 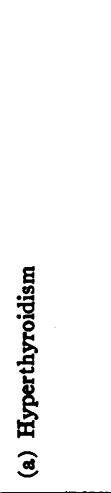 & 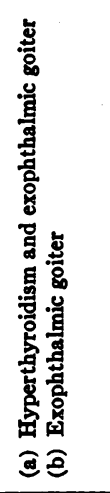 & 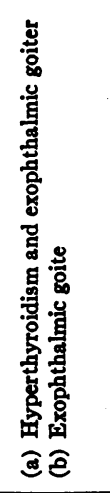 & 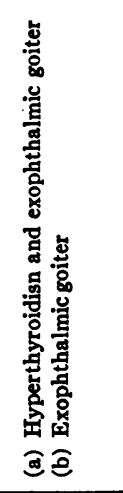 & 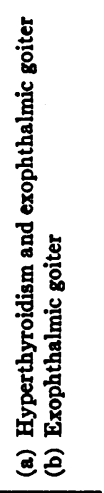 \\
\hline 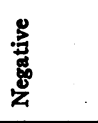 & 葍造 & 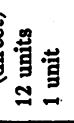 & 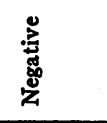 & 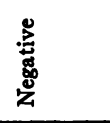 & 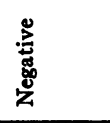 & 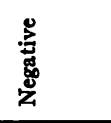 & 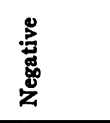 & 总 & 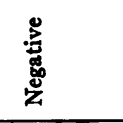 & $\underset{\mathbb{E}}{\stackrel{\rightleftarrows}{\sharp ~}}$ \\
\hline 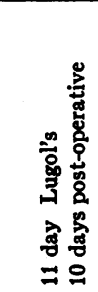 & 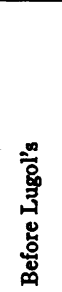 & 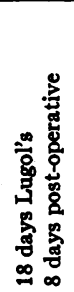 & 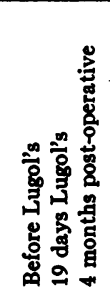 & 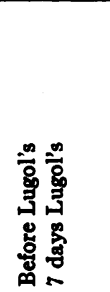 & 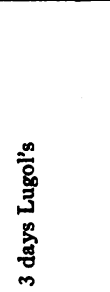 & 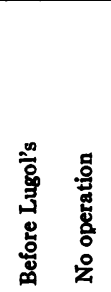 & 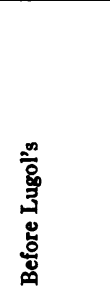 & 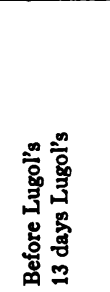 & 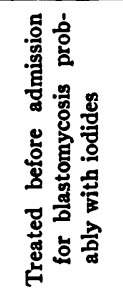 & 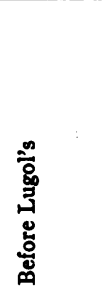 \\
\hline $\overrightarrow{+}+\frac{1}{4}$ & & $\overrightarrow{\tilde{t}}$ & 孚夲壱 & 安望 & $\stackrel{\mathscr{n}}{+}$ & 守 & ๘্ & 㐫尔 & 苂 & क्षे \\
\hline 范。 & in & in & G9mo & $\overrightarrow{0} 0$ & is & $\overrightarrow{0}$ & $\stackrel{\square}{n}$ & Ti & $\stackrel{\infty}{+}$ & $\stackrel{\infty}{+\infty}$ \\
\hline 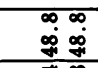 & $\stackrel{5}{4}$ & 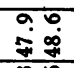 & 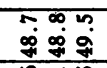 & $\begin{array}{l}\infty \\
\dot{g} \\
\dot{q}\end{array}$ & $\begin{array}{l}\infty \\
\infty \\
\infty \\
j\end{array}$ & ị & $\begin{array}{l}0 \\
9\end{array}$ & कूँ & ma & $\begin{array}{l}\infty \\
\infty \\
\infty \\
0\end{array}$ \\
\hline $\begin{array}{l}+\infty \\
\dot{m} \\
\dot{n}\end{array}$ & $\stackrel{a}{i}$ & 虫 & 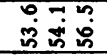 & 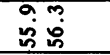 & i & $\vec{p}$ & : & कृ & $\stackrel{m}{m}$ & ن. \\
\hline 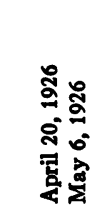 & 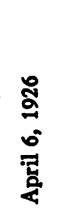 & 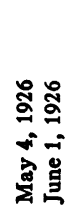 & 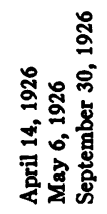 & 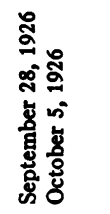 & 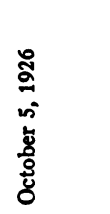 & 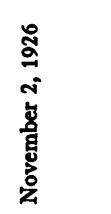 & 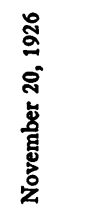 & 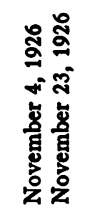 & 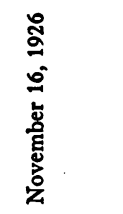 & 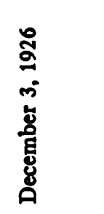 \\
\hline 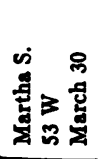 & di & 疍 & 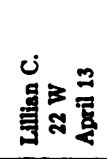 & 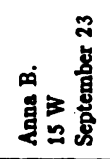 & 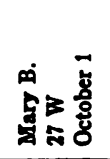 & 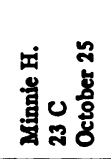 & 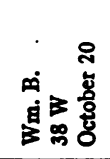 & 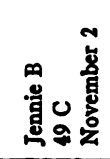 & 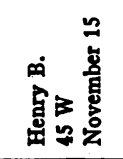 & 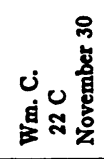 \\
\hline$\vec{\sim}$ & $\mathbb{N}$ & & $\approx$ & ন & $\dddot{\alpha}$ & $\stackrel{\sim}{ }$ & $\approx$ & జి & ని & 요 \\
\hline
\end{tabular}




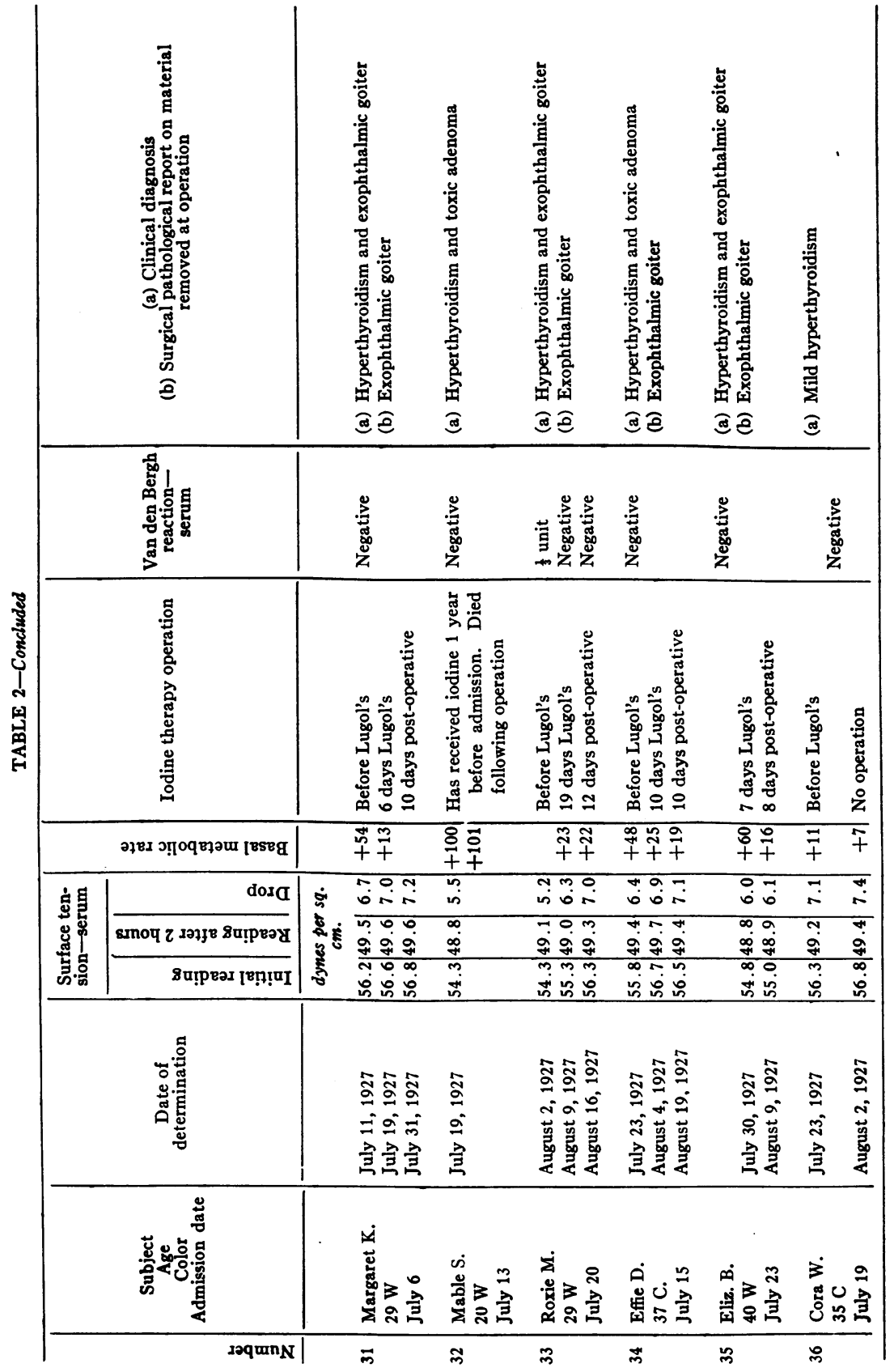


E. G. NICHOLLS AND G. A. HARROP, JR.

\begin{tabular}{|c|c|c|c|c|c|c|c|}
\hline 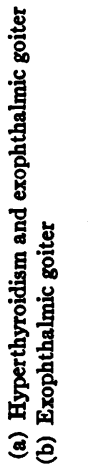 & & 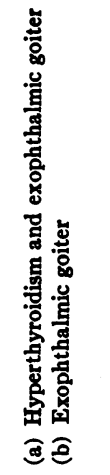 & 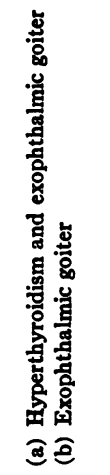 & 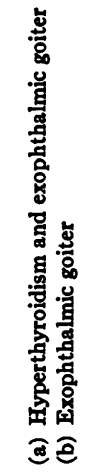 & 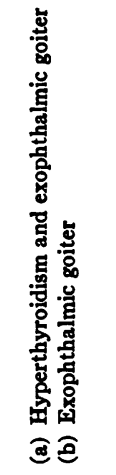 & 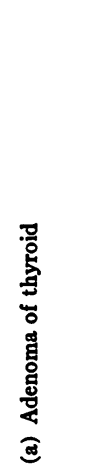 & 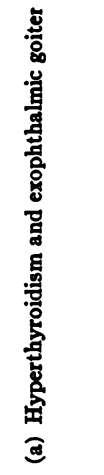 \\
\hline 总 & 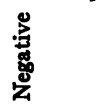 & 芯 & 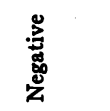 & 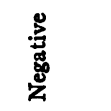 & 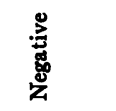 & 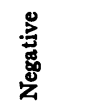 & 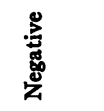 \\
\hline 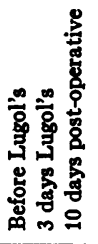 & 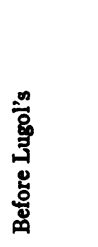 & 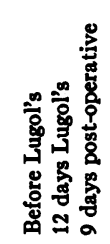 & 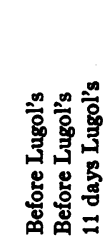 & 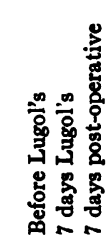 & 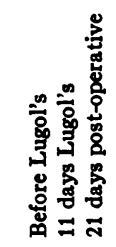 & 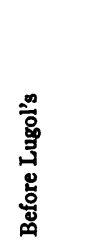 & 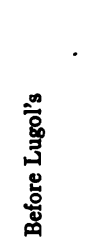 \\
\hline 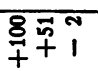 & $\begin{array}{l}8 \\
+\end{array}$ & $\overrightarrow{\mathrm{p}} \stackrel{\mathrm{m}}{+}$ & 导算䎡 & $\begin{array}{l}\vec{m}+N \\
+++\end{array}$ & 可年 & $\overrightarrow{+}$ & $\stackrel{\infty}{+}$ \\
\hline तु mo & $\stackrel{\infty}{+\infty}$ & Ho no & : & พִ m? & फ़造 & $\stackrel{2}{n}$ & $\stackrel{5}{*}$ \\
\hline 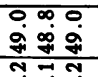 & $\frac{\dot{0}}{\dot{g}}$ & $\begin{array}{l}9 \\
g \\
g\end{array}$ & 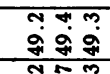 & $\begin{array}{l}\infty \\
g \\
g\end{array}$ & 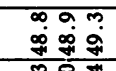 & $\ddot{g}$ & $\frac{\pi}{9}$ \\
\hline ஸे ఉં & 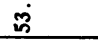 & 象出 & ค & is & 出 & $\ddot{\varphi}$ & 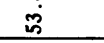 \\
\hline 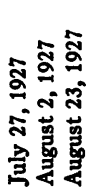 & 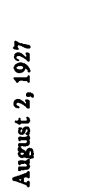 & 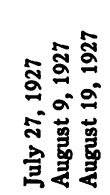 & 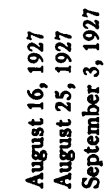 & 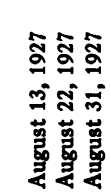 & 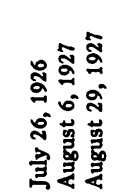 & 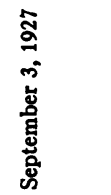 & 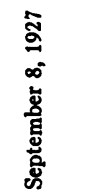 \\
\hline 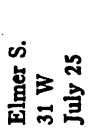 & 焉瓷 & 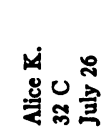 & 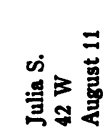 & 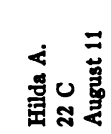 & 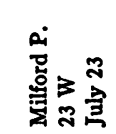 & 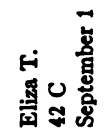 & 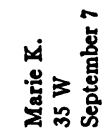 \\
\hline & ల & & 5 & & & $=$ & \\
\hline
\end{tabular}


minimal. Serum was also preferred to plasma because of the danger of hemolysis when an anti-coagulant was used. Plasma was found to give a parallel but slightly higher reading than serum. This was true in the case of oxalated blood and also when coagulation was prevented by a highly purified sample of heparin, kindly supplied to us by Dr. W. H. Howell.

The surface tension of the blood serum of forty-four patients suffering with hyperthyroidism was determined in the above way and, for comparison, that of fourteen normal individuals and twelve persons with miscellaneous conditions. Table 1 gives the data obtained from the study of the controls, and table 2 the data obtained from that of the cases of thyroid intoxication.

\section{DISCUSSION}

From table 1 it will be seen that the initial surface tension in the normal cases varied between 56.6 and $\mathbf{5 7 . 7}$ dynes, and the two-hour time drop varied between 6.9 and 8.2 dynes. From table 2 it will be noted that thirty-nine out of forty-four cases of thyroid intoxication previous to treatment showed an initial surface tension reading of 56.5 dynes or less and that the time drop in thirty-eight of these cases was less than the minimal drop in the normal controls. These relationships are shown graphically in chart 1.

We consider that this data furnishes substantial evidence that during the period of thyroid intoxication a surface active substance is usually if not invariably present in the blood serum. Such a lowering of the surface tension in thyroid disease could be accounted for by the presence of bile acids in the serum. Since no convenient method was available at the time for the estimation of the bile salts the Van den Bergh reaction was carried out in all but seven of the cases to serve as an indirect indication of the presence of bile. Of the thirtyseven cases thus studied an amount of bilirubin above the normal limits, a "positive" Van den Bergh reaction, was found in the serum of four. Two of the cases with a "positive" Van den Bergh showed no jaundice clinically and only a trace of bilirubin in the serum. Serum from another case showed $\frac{1}{3}$ unit of bilirubin on admission but the Van den Bergh reaction was negative after nineteen days treatment with Lugol's solution. Nevertheless the lowered surface tension 


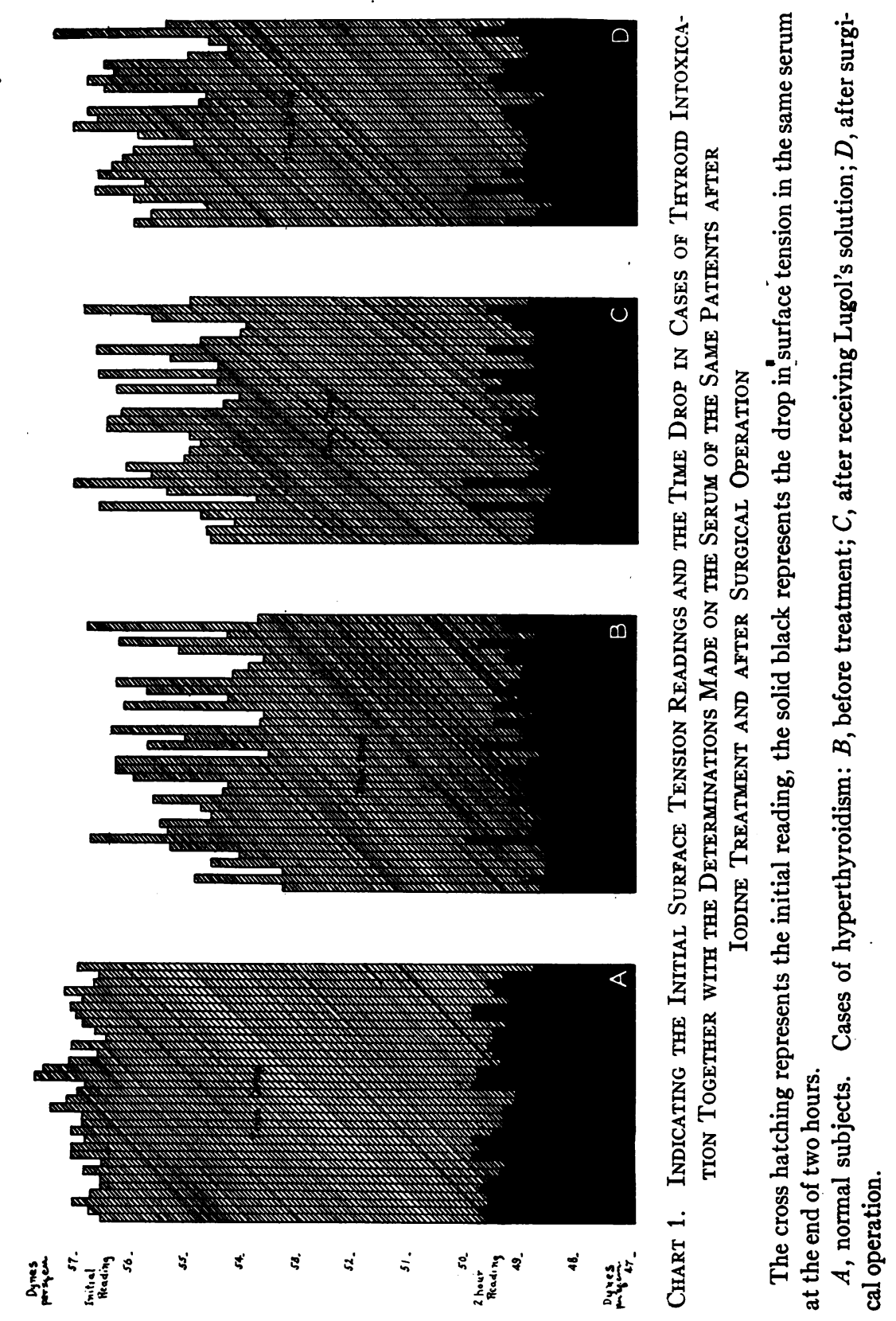


of the serum still persisted at this time. Of the seven cases in which the Van den Bergh reaction was not made, two showed slight icteric discoloration of the sclerae on admission which had cleared up at the time that the surface tension studies were made. The other five cases showed no clinical evidence of jaundice. All of these observa.. tions are indicated in table 2. Aside from these exceptions none of the cases showed either clinical evidence of jaundice or an abnormal amount of bilirubin in the blood serum as indicated by the Van den Bergh reaction.

We have considered the possibility that the surface active substance which appears to be present may be an unsaturated compouna, possibly an unsaturated fatty acid. This aspect is further dealt with in the following paper (4). Under such circumstances the administration of iodine, even in the rather small amounts of Lugol's solution which are effective therapeutically, might be sufficient to neutralize its effect. However, the fact that iodine administration has not markedly altered the low surface tension of the serum in this condition appears to indicate that such is not the explanation of its action. After partial thyroidectomy it also appears that the return of the surface tension of the serum to normal is very slow.

No previous report has been found of a lowering of the surface tension of the blood serum in thyroid disease. Adlersburg and Sugär (2) state that the surface tension of the urine is lowered in Basedow's Disease. While the present study was in progress a paper by Wilhelmj and Fleisher (3) appeared, in which they reported that after thyroidectomy in guinea pigs a gradual rise in the surface tension of the plasma occurred, so that in nineteen to twenty-two days the readings were definitely abnormal. They find that the time drop, after twenty minutes, was in general less in the plasma from the animals operated upon than in the plasma from normal controls. Essentially the same differences were found by them when readings were made after two hours as were found after an interval of twenty minutes. These authors also made a study of the effect of thyroxin and thyroid extract administration to guinea pigs. In most cases a definite decrease in the surface tension of the plasma was found, but little change on the average was detected in the time drop. 


\section{CONCLUSIONS}

1. The surface tension of the blood serum is lower than normal in many cases of thyroid intoxication. This is associated with a diminished time drop at the end of two hours, when compared with that present in the serum of normal persons.

2. The administration of iodine in the form of Lugol's solution has an appreciable effect in increasing the lowered surface tension.

3. After operation involving the partial removal of the thyroid gland there is a tendency for the surface tension of the serum to rise even more than after iodine.

\section{BIBLIOGRAPHY}

1. Du Noüy, P. Lecomte, J. Exp. Med., 1922, xxxv, 575. Spontaneous Decrease of the Surface Tension of Serum. I.

Du Noüy, P. Lecomte, J. Exp. Med., 1923, xxxviii, 87. Surface Tension of Serum. VI. The Study of Immune Serum, Time-Drop and Initial Value of Surface Tension.

Du Noüy, P. Lecomte, J. Exp. Med., 1924, xxxix, 37. Surface Tension of Serum. VII. Significance of the Maximum Time-Drop of Serum Solutions.

Du Noüy, P. Lecomte, J. Gen. Physiol., 1924, vi, 625. Surface Tension of Serum. XI. An Improvement of the Technique for Measuring Surface Tension.

2. Adlersberg, D., and Sugár, M., Ztschr. f. d. ges. exper. Med., 1925, xlvi, 466. Über den Tagesablauf der Oberflächenspannungsstudien im Harn bei pathologischen zuständen.

Adlersberg, D., Wien. klin. Wchnschr., 1925, xxxviii, 1051. Ueber die Ober-

- flächenspannung von Körperflüssigkeiten bei normalen und pathologischen Zuständen.

3. Wilhelmj, C. M., and Fleisher, M. S., J. Exper. Med., 1926 xliii, 179 and 195. The Relation of the Thyroid Gland to the Surface Tension of the Blood Plasma. I and II.

4. Nicholls, E. H., and Perlzweig, W. A., J. Clin. Invest., 1928, v, 195. The Plasma Fats and the Iodine Absorption Capacity of the Fatty Acids in Hyperthyroidism. 